\title{
Comprehensive review of the incisive branch of the inferior alveolar nerve
}

\author{
Jennifer A. Caughey ${ }^{1}$, Quang $\mathrm{Do}^{2}$, Daniel Shen ${ }^{2}$, Hiroe Ohyama ${ }^{3}$, Puhan $\mathrm{He}^{1}$, R. Shane Tubbs ${ }^{4,5,6,7,8,11}$, \\ Joe Iwanaga ${ }^{4,5,9,10}$ \\ ${ }^{1}$ Department of Oral and Maxillofacial Surgery, University of Pennsylvania, Philadelphia, PA, ${ }^{2}$ Harvard School of Dental Medicine, Harvard University, \\ Boston, MA, ${ }^{3}$ Department of Restorative Dentistry and Biomaterials Sciences, Harvard School of Dental Medicine, Boston, MA, ${ }^{4}$ Department of \\ Neurosurgery, Tulane Center for Clinical Neurosciences, Tulane University School of Medicine, New Orleans, LA, ${ }^{5}$ Department of Neurology, Tulane \\ Center for Clinical Neurosciences, Tulane University School of Medicine, New Orleans, LA, USA, ${ }^{6}$ Department of Anatomical Sciences, St. George's \\ University, St. George's, Grenada, ${ }^{7}$ Department of Structural \& Cellular Biology, Tulane University School of Medicine, New Orleans, LA, ${ }^{8}$ Department \\ of Neurosurgery and Ochsner Neuroscience Institute, Ochsner Health System, New Orleans, LA, USA, ${ }^{9}$ Dental and Oral Medical Center, Kurume \\ University School of Medicine, Kurume, Fukuoka, ${ }^{10}$ Division of Gross and Clinical Anatomy, Department of Anatomy, Kurume University School of \\ Medicine, Kurume, Fukuoka, Japan, ${ }^{11}$ Department of Surgery, Tulane University School of Medicine, New Orleans, LA, USA
}

\begin{abstract}
The incisive branch of the inferior alveolar nerve is a vital anatomical structure within the anterior mandible that has not been thoroughly defined and outlined in reports in the literature until recent years. Advances in radiological imaging, particularly the widespread use of cone-beam computed tomography has allowed for accurate visualization of the mandibular incisive canal (MIC) and its associated incisive branch of the inferior alveolar nerve. Surgical damage to the MIC, which could result in hemorrhage and sensory disturbance, may occur in commonly practiced oral and maxillofacial procedures, such as chin bone harvesting, implant placement, fracture repair and removal of pathologic entities of the anterior mandible. Knowledge of both the presence, dimensions and location of the incisive branch is a vital component to pre and peri-operative planning of oral and maxillofacial surgeries performed within the mandible, particularly within the interforaminal zone. In this article, the terminology, anatomy, imaging, surgical consideration, and pathology of the incisive branch will be discussed.
\end{abstract}

Key words: Mandible, Incisive canal, Dental implants, Oral surgery, Cone-beam computed tomography

Received June 2, 2021; Revised July 1, 2021; Accepted July 13, 2021

\section{Introduction}

The inferior alveolar nerve (IAN) is a sensory branch of the mandibular division of the trigeminal nerve, providing innervation to the chin, lower lip, mandibular teeth and buccal (labial) gingiva of the anterior teeth. Traditionally, the IAN has been described as a single trunk that courses through the mandibular canal until it splits into its major

\footnotetext{
Corresponding author:

Joe Iwanaga (i)

Department of Neurosurgery, Tulane Center for Clinical Neurosciences, Tulane University School of Medicine, New Orleans, LA 70112, USA E-mail: iwanagajoeca@gmail.com
}

terminal branches, the mental nerve and incisive branch, prior to exiting the mental foramen (Fig. 1) [1-3]. The mental nerve then exits the mandibular canal through the mental foramen, while the incisive branch continues through the anterior mandible in the mandibular incisive canal (MIC) [4]. The incisive branch then continues within the MIC or constitutes the incisive plexus, providing innervation to the premolar, canine, and incisor teeth and associated gingiva [5].

The presence and location of the incisive branch of the IAN is an important consideration for both anesthetic technique and surgical planning within interforaminal zone, which includes the mandibular incisors, canines and first premolars. The interforaminal zone has generally been considered a safe location for implant placement, as well as an ideal donor site for chin block bone grafting, due to its ideal

\section{Copyright ( 2021 . Anatomy \& Cell Biology}

This is an Open Access article distributed under the terms of the Creative Commons Attribution Non-Commercial License (http://creativecommons.org/licenses/by-nc/4.0/) which permits unrestricted non-commercial use, distribution, and reproduction in any medium, provided the original work is properly cited. 


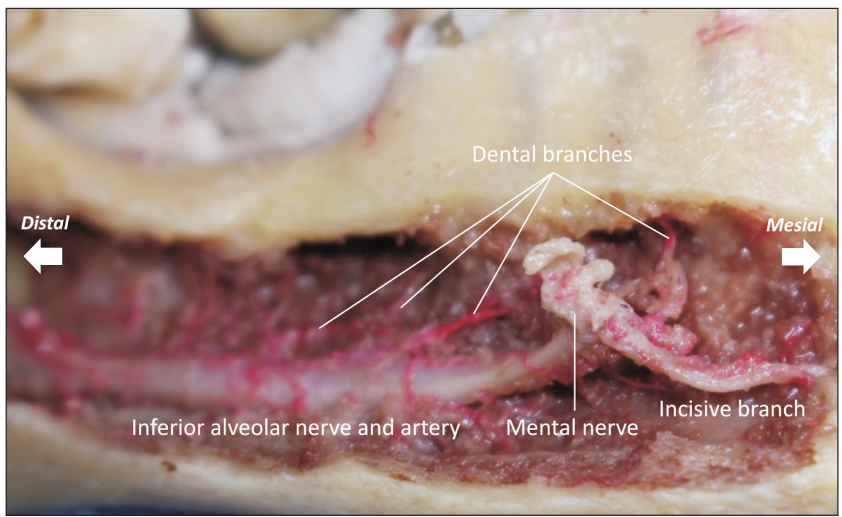

Fig. 1. Cadaveric example of the incisive branch of the inferior alveolar nerve and artery. Note the buccal cortical plate of the mandible is removed (right side).

bone quality and distance from the IAN in comparison to the posterior mandible [6]. However, careful imaging of associated neurovascular bundles within the interforaminal zone is still essential as a means of preventing surgical complications, such as sensory alterations in the anterior teeth, skin, and mucosa. Despite the clinical importance of knowledge of the location of the neurovascular bundles and associated structures within the interforaminal zone, there are varying clinical guidelines regarding the optimal location for procedures such as implant placement and bone graft harvest [6].

This review will examine the various imaging techniques used to identify and analyze the incisive branch of the IAN, as well as discuss the common anatomical variations, including frequency of appearance, dimensions and location. Implications of these findings will be discussed in relation to both clinical application and associated pathologies.

\section{Review}

\section{Terminology}

Interestingly, the incisive branch of the IAN is not included in Terminologia Anatomica published by the Federative International Programme for Anatomical Terminology (FIPAT), which prescribes international anatomical terminology.

The incisive branch of the IAN has been previously documented under several different names, such as the lingual portion of the anterior mandibular canal, the MIC, incisive canal, incisive nerve canal, the mandibular incisive nerve, as well as the incisive bundle or incisive plexus [6, 7]. In regards to the occurrence and prevalence of the incisive branch, the majority of the literature has relied upon documentation of the MIC and subsequently, its housing of the incisive branch itself, as the MIC has now been widely accepted as a significant anatomical finding [8] despite previous studies having neglected its occurrence [9]. The MIC, with its contained incisive branch, is alternatively referred to as the mandibular incisive nerve and is rarely documented under alternative nomenclature, such as the lingual portion of the anterior mandibular canal as stated above.

\section{Anatomy}

\section{Blood supply}

The inferior alveolar artery travels with the IAN through the mandible within the mandibular canal. After reaching the interforaminal region, the mandibular canal splits into the MIC and mental canal, with the MIC continuing along the anterior mandible, containing vascular bundles that supply the incisors, canines, mandibular first premolars and associated attached mucosa $[10,11]$. Jacobs et al. [12] observed through utilization of high-resolution magnetic resonance imaging (MRI), that the MIC contains its own true neurovascular bundle, as the intraosseous extension of the inferior alveolar neurovascular bundle [13]. Additionally, some have considered that, while the MIC may become indistinct at the level of the central incisors, a neurovascular plexus may exist at the mandibular midline [7].

\section{Innervation}

Traditionally, the incisive branch innervates the ipsilateral mandibular premolars, canines and incisors $[1,11]$. However, there have been several reported anatomical variations of the incisive branch that may lead to subsequent deviations from the traditional structural innervation [7]. It has been demonstrated that the incisive branch itself is extensively branched and can cross the midline to innervate the contralateral side of the mandible [14]. It has also been observed that the midline of the body of the mandible may not correspond with the midline of the incisive branch [15]. Additionally, there is considerable variation on which mandibular teeth contain communication between both the mental nerve and incisive branch [1].

\section{Size and location}

The precise location of the bifurcation of the IAN into the mental nerve and incisive branch may vary (Table 1, Fig. 2) 
Table 1. Diameter and length of MIC

\begin{tabular}{|c|c|c|c|}
\hline Author & Study material & Diameter of MIC at specific site & Length of MIC \\
\hline Mardinger et al. [16] & $\begin{array}{l}20 \text { cadaveric mandibles and } \\
6 \text { hemimandibles }\end{array}$ & $\begin{array}{l}2.09 \pm 0.42 \mathrm{~mm} \text { at the mental canal, } 1.25 \pm 0.24 \mathrm{~mm} \text { near the position } \\
\text { of the canines, and } 0.98 \mathrm{~mm} \text { near the lateral incisors }\end{array}$ & - \\
\hline Parnia et al. [17] & $96 \mathrm{CBCT}$ radiographs & $\begin{array}{l}4.46 \pm 1.40 \mathrm{~mm} \text { from the lingual border, } 3.48 \pm 1.17 \mathrm{~mm} \text { from the } \\
\text { buccal border, and } 8.72 \pm 1.43 \mathrm{~mm} \text { from the lower border }\end{array}$ & - \\
\hline Gilis et al. [18] & СВCT of 100 hemimandibles & 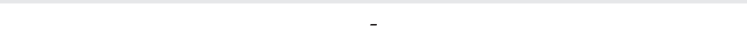 & $6.65 \pm 4.1 \mathrm{~mm}$ \\
\hline Kong et al. [19] & СBCT of 100 hemimandibles & $\begin{array}{l}\text { Horizontal diameter at origin: } 2.16 \pm 0.58 \mathrm{~mm} \text {, vertical diameter at } \\
\text { origin } 2.15 \pm 0.62 \mathrm{~mm} \\
\text { Horizontal diameter at end: } 0.84 \pm 0.23 \mathrm{~mm} \text {, vertical diameter at end: } \\
0.89 \pm 0.34 \mathrm{~mm}\end{array}$ & $\begin{array}{l}17.84 \mathrm{~mm} \text {, left mandible } \\
17.73 \mathrm{~mm} \text {, right mandible }\end{array}$ \\
\hline
\end{tabular}

MIC, mandibular incisive canal; CBCT, cone-beam computed tomography.

[16-19]. For example, the mental nerve may turn superiorly and inferiorly to the location of the bifurcation to exit the mental foramen, forming an anterior loop $[4,20]$. The incisive branch and associated MIC travel through the intramedullary space, with the MIC usually located medial to the mental foramen, with a narrowing canal diameter and less cortical bone as it approaches the anterior mandible [9]. The length of the incisive branch has also been suggested to grow in association with loss of mandibular dentition [11].

In an examination of 46 hemi-mandibles, Mardinger et al. [16] demonstrated the mean incisive canal diameter to be 2.09 $\mathrm{mm}$ at the mental canal, $1.25 \mathrm{~mm}$ near the position of the canines and $0.98 \mathrm{~mm}$ near the lateral incisors. In a study of 96 cone-beam computed tomography (CBCT) radiographs taken in anticipation of implant placement conducted by Parnia et al. [17], the diameter of the incisive canal was reported to be $1.47 \pm 0.50 \mathrm{~mm}$. The mean distance of incisive canal from the borders of the mandible were found to be as follows: $4.46 \pm 1.40 \mathrm{~mm}$ from the lingual border, $3.48 \pm 1.17 \mathrm{~mm}$ from the buccal border and $8.72 \pm 1.43 \mathrm{~mm}$ from the lower border.

Gilis et al. [18] utilized CBCT to examine the presence, positioning and diameter of the MIC in transverse imaging of 100 hemimandibles. When visualized, the MIC was found to course in a descending manner toward the mandibular symphysis. The average length of the MIC in all groups was found to be $6.65 \pm 4.1 \mathrm{~mm}$. A significantly larger horizontal diameter and length of the MIC was demonstrated in edentulous patients and patients over the age of 60 years. Additionally, the MIC was found to be more superficial to the alveolar ridge in this patient population.

Kong et al. [19] obtained 50 CBCT, or 100 hemimandibles, to measure the MIC diameter, length and location within the mandible and found that the diameter of the MIC gradually decreased from origin, from the area of the second premolar tooth, to end, near the central incisor teeth. The horizontal

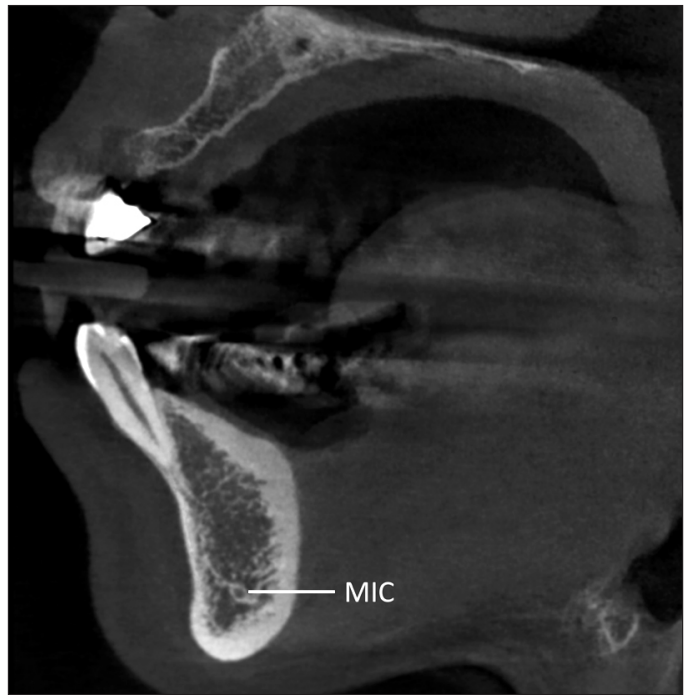

Fig. 2. Mandibular incisive canal (MIC) in the lower canine region in sagittal cone-beam computed tomography. Note the MIC is surrounded by the dense bone.

diameter at the origin measured $2.16 \pm 0.58 \mathrm{~mm}$ and the vertical diameter measured $2.15 \pm 0.62 \mathrm{~mm}$, compared to the horizontal diameter of $0.84 \pm 0.23 \mathrm{~mm}$ and vertical diameter of $0.89 \pm 0.34 \mathrm{~mm}$ at the end. The authors additionally demonstrated that the distances from the MIC to the lower margin of the mandible at the second premolar and first premolar teeth differed significantly according to sex, with the mean distance from the MIC to the lower margin at the second premolar measuring $11.34 \mathrm{~mm}$ in males and $8.75 \mathrm{~mm}$ in females. Similarly, the mean distance at the first premolar was $9.44 \mathrm{~mm}$ in males and $8.43 \mathrm{~mm}$ in females. The mean length of the MIC was found to be $17.84 \mathrm{~mm}$ in the left side of the mandible and $17.73 \mathrm{~mm}$ in the right side of the mandible. 


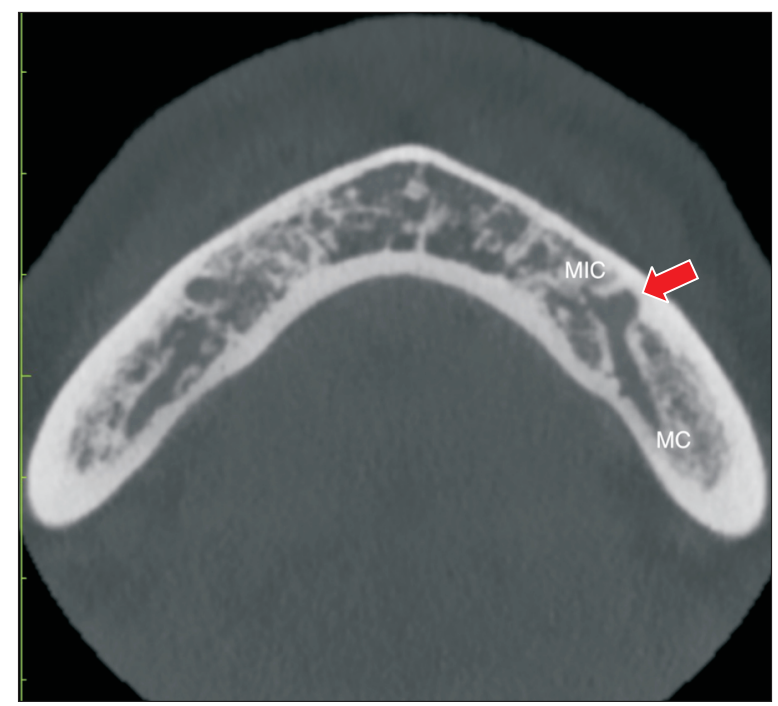

Fig. 3. Continuity of the mandibular incisive canal (MIC) and mandibular canal (MC) shown in axial cone-beam computed tomography. The bony canal to the mental foramen is also shown (arrow).

\section{Incidence in cadaveric studies and different imaging modalities}

Several types of imaging modalities have been employed to analyze the incisive branch of the IAN, including cadaveric studies, panoramic radiographs, and CBCT (Fig. 3).

Cadaveric studies have been utilized both as means of direct visualization of the course of the incisive branch of the IAN, as well as to observe the macroscopic content of the MIC. The buccal cortical plate often requires removal before a grinding apparatus can be used to the expose the entire course of the IAN (Fig. 1). The position, length, and diameter of IAN and its associated anatomical structures can then be measured with a digital-sliding caliper [4]. The presence of the incisive branch of the IAN has been detected $100 \%$ of the time in several cadaveric studies $[4,15,21]$. While cadaveric studies allow for a better understanding of the presence and anatomical variations of the incisive branch among different populations, these studies alone may be less applicable when applied to a clinical setting. Additional studies have also applied traditional radiographic techniques or $\mathrm{CBCT}$ on cadaveric mandibles to further compare dissection and radiographic findings, allowing for a greater understanding of the reliability of the employed radiographic images $[15,21]$.

Traditional radiographs, such as periapical radiographs, have been used to evaluate the incisive branch; however, these types of radiographs, while efficient and typically taken at the majority of initial dental examinations, do not

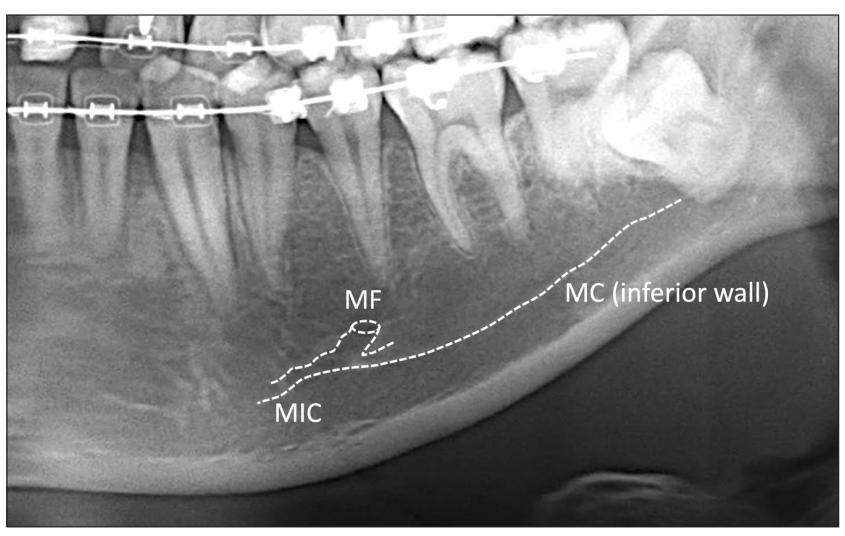

Fig. 4. Mandibular incisive canal (MIC) in panoramic radiograph. Note the initial part of the MIC is visible but not clear mesial to the first premolar. MF, mental foramen; MC, mandibular canal.

alone provide enough accurate information for treatment planning purposes. There has been no correlation found between the incisive branch's anatomical and radiographical findings when utilizing traditional radiographs. This may be attributed to the distortion often associated with radiographic films and is further complicated by variations in the size and uniformity of the bone trabeculae surrounding the MIC $[1,16]$.

Panoramic radiographs, while commonly utilized as a means of assessing dentition, jawbones, sinuses, and the temporomandibular joints, provide limited information on the incisive branch. Due to the confines of two-dimensional imaging, panoramic radiographs do not display the buccolingual aspect and cross-sectional slices of the MIC and incisive branch. Panoramic radiographs are additionally highly dependent on patient positioning, which may lead to magnification, distortion, and superimposition of anatomical structures. Notably, Jacobs et al. [12] demonstrated that the MIC was only identified in $15 \%$ of panoramic radiographs of 545 patients, and clearly visible in only $1 \%$ of cases (Fig. 4). A later study by Pires et al. [22] reported similar findings, demonstrating that panoramic radiographs accurately detected the MIC in only $11 \%$ of patients. Sahman et al. [23] showed that the MIC was visible $51.2 \%$ of panoramic radiographs, however; this group also established that the size of the MIC did not significantly affect its visibility, suggesting that even anatomically large MICs may not be visible in panoramic imaging.

CBCT may provide multiplanar views of high-resolution images at reduced radiation doses when compared to MRI and CT. Consequently, CBCT has been found to be a practi- 
cal means of detecting and evaluating the incisive branch of the IAN. Sahman et al. [23] demonstrated that the MIC was visible in $94.4 \%$ of CBCT radiographs, compared to $51.2 \%$ of panoramic radiographs. Pereira-Maciel et al. [24] found that the MIC can be detected bilaterally in $100 \%$ of CBCT radiographs. If available, CBCT should be employed as the radiographic imaging modality of choice when planning for any type of surgical procedure involving the anterior mandible given its demonstrated high degree of accuracy in the display of the MIC.

\section{Surgical considerations}

\section{Bone graft}

Autologous bone grafts harvested from the mandibular symphyseal region have traditionally been employed as an ideal site for bone harvesting due to ease of access, bone type and quality. Previous groups have emphasized the importance of creating osteotomy lines at least $5 \mathrm{~mm}$ from the root apices of teeth to ensure preserved teeth vitality [7, 8]. The general recommended safety margin, as outlined by Hunt \& Jovanovic, is for the harvest zone to be $5 \mathrm{~mm}$ anterior to the mental foramen, $5 \mathrm{~mm}$ below the tooth apices and $5 \mathrm{~mm}$ above the lower border of the mandible [25].

The incisive branch has increasingly been recognized as an important anatomical structure when harvesting bone from the interforaminal region due to the risk of neurosensory deficit [26]. Sbordone et al. [27] assessed neurosensory disturbances and tooth-pulp sensitivity loss following mandibular parasymphyseal bone harvesting, leaving $5 \mathrm{~mm}$ of space between the foramina, dental root apices and basal bone when creating osteotomy lines. This group found that when the osteotomy line was within $5 \mathrm{~mm}$ of the root apices, $39 \%$ of teeth lost pulp sensitivity. Of note, $33 \%$ of teeth lost vitality even when the osteotomy was $>5 \mathrm{~mm}$ from the teeth apices, lending to the conclusion that loss of pulpal sensitivity may not be correlated to the distance between the root apex and harvested bone. This may be attributed to the anatomical variability of the MIC in the buccal-lingual direction.

Pommer et al. [28] found that applying the generally recommended safety margins during symphyseal bone graft harvesting allowed for a significant risk of nerve damage and provided a new set of recommendations, concluding that symphyseal bone should be harvested at least $8 \mathrm{~mm}$ below the tooth apices, with a maximum harvest depth of
$4 \mathrm{~mm}$. Kong et al. [19] found the mean distances from the MIC to the tooth apex at the first premolar, canine, lateral incisor and central incisor to be over $7.74 \mathrm{~mm}$ and the mean distances from the MIC to the lower margin to be over 8.21 $\mathrm{mm}$. Based upon these measurements, the authors concluded that a chin bone graft could safely be harvested above or below the MIC. Additionally, because the MIC was found to be in close proximity to the buccal cortical border, with the longest distance measuring $4.65 \mathrm{~mm}$, the authors suggested that harvesting depth should be limited to $4 \mathrm{~mm}$.

Al-Ani et al. [26] identified the MIC on sixty CBCT examinations and found the median distance of the MIC from the lower border of the mandible to be $9.86 \pm 2.51 \mathrm{~mm}$. This group contrarily concluded that the traditional harvest zone safety margin as described by Hunt and Jovanovic is a safe zone for bone harvesting from the interforaminal region of the mandible and should be employed in centers when CBCT may not be available. The MIC was visible on $100 \%$ of CBCTs taken, further demonstrating the necessity of CBCT for preoperative planning.

\section{Implant surgery}

Though the mandibular interforaminal zone has traditionally been considered a low-risk area for implant placement, complications may arise from damage to the MIC and the associated incisive branch, such as hemorrhage and neurosensory disturbance [29, 30]. Lee et al. [29] demonstrated a case of hemorrhage from laceration of the vascular structures contained within the incisive canal during implant osteotomy placement. Kütük et al. [31] conducted a retrospective study to evaluate neuropathic pain caused by implant placement in the interforaminal region of the mandible in relation to the MIC and found that, of the 10 patients that were evaluated for neurosensory disturbances, radiographic imaging confirmed at least 1 MIC perforation by an implant in each patient.

When placing implants in the interforaminal zone, the diameter and direction of the MIC should be verified during the initial treatment planning stages. Drilling during implant placement may lead to injury of the incisive branch, particularly in the case of an incisive branch with a large diameter. This can subsequently lead to stretching of and further damage to the IAN [11]. Patients with a large MIC may be more prone to experiencing higher amounts of intraoperative and post-operative pain, which may even necessitate implant removal [32]. 
The MIC in elderly and edentulous patients has been found to follow a more internalized and superficial course in relation to the overlying alveolar ridge, making the incisive branch more vulnerable to damage during implant placement in the interforaminal zone. Wismeijer et al. [33] demonstrated permanent sensory deficits in the lower lip in $7 \%$ of 110 edentulous patients who received 2 to 4 implants in the interforaminal zone, with each implant placed at least 3 $\mathrm{mm}$ away from the mental foramen. It has also been reported that, since the average diameters of the MIC at its origin have been reported to be around $2 \mathrm{~mm}$, overgrowth of connective tissue may prevent implant osteointegration should the implant be placed in a 2-mm cavity [18].

Identification of the MIC by CBCT prior to implant placement should be employed whenever possible as part of the pre-operative planning to assist in avoiding iatrogenic damage to the incisive branch.

\section{Fracture}

Mandibular fractures may result in injury to the IAN, with subsequent sensory disturbances occurring to the lower lip and chin. A review of studies that evaluated neurosensory disturbances following mandibular fractures found that the reported IAN sensory deficit after injury before treatment was $5.4 \%-81.4 \%$, shortly following treatment was $0.4 \%-$ $91.3 \%$ and after 1 year was $0 \%-46.6 \%$ [34]. Most studies reporting on nerve injuries following mandible fractures and subsequent repair do not specifically focus on damage to the incisive branch and MIC; however, it has been documented that fractures of the IAN-containing posterior mandible are associated with higher risk of both pre- and postoperative sensory disturbances when compared to non-IAN bearing sites. Tay et al. [34] demonstrated that the prevalence of IAN injury in IAN bearing sites was higher by $43.6 \%$ before treatment and $41.2 \%$ after treatment when compared to nonIAN bearing sites. This group posed that the presence of preoperative sensory alteration in the anterior mandible may be related to post-traumatic swelling and that post-operative sensory alteration following open reduction-internal fixation (ORIF) may be explained by plating in close proximity to the mandibular foramen.

Displaced fractures of the anterior mandible in the area of the incisive branch and MIC, specifically parasymphyseal and symphyseal fractures, are traditionally treated with ORIF [35]. Two miniplates or one larger plate have generally been accepted as the ideal treatment method. It should be noted that the use of two mini plates has been associated with more postoperative complications, such as infection, wound dehiscence and iatrogenic damage to tooth roots [36]. Two lag screws that span the fracture line may also be used as means of fixation; however, this technique has been associated with reported paresthesia in the mental nerve region of up to $68 \%$ of cases, and can be considered to be more technique sensitive [37]. Careful attention to the positioning of the IAN, mental foramen and MIC should be evaluated prior to fracture repair via $\mathrm{CBCT}$ if available and during the repair of mandibular fractures to minimize the risk of iatrogenic nerve damage.

\section{Cystectomy}

There are numerous types of intra-bony lesions that may present as a unilocular lesion of the tooth-bearing portions of the anterior mandible, such as keratocystic odontogenic tumor, radicular cyst, glandular odontogenic cyst, and adenomatoid odontogenic tumor. Central mucoepidermoid carcinoma, a low-grade malignancy, is less likely, but may present as a well-demarcated lesion near the mandibular midline. Developmental entities, such as an anterior Stafne bone defect, may also present in a similar fashion in the anterior mandibular region [38]. Careful attention should be payed to the positioning and presence of the MIC and incisive canal in relation to identification and treatment of anterior mandibular and periapical pathology.

Bilginaylar et al. [39] described the enucleation of a radicular cyst along the area of the left mandibular premolar and subsequent exposure of the incisive branch. Following the exposure, the authors rinsed the cystic cavity with sterile physiologic saline and placed platelet-rich fibrin gel inside the bony defect. The patient did display post-operative paresthesia, which completely resolved after two months. Similar to the above mentioned surgical procedures, the presence, location and dimensions of the MIC should be elicited when planning for the biopsy or removal of any pathology present in the anterior mandible.

\section{Pathology}

Primary bone tumor associated with neural tissue, such as schwannomas, in the anterior mandible is extremely rare [40]. The analysis of 71 cases showed that the majority of the central schwannomas involved with posterior mandible (78\%) and only $22 \%$ was associated with the anterior mandible [40]. Rengaswamy could not find the relationship between the 
schwannoma and IAN, and suggested that the origin of the tumor could have been one of the branches of the incisive branch [41].

\section{Conclusion}

The incisive branch of the IAN is an important anatomical consideration for both pre and peri-operative planning for oral and maxillofacial surgical procedures conducted in the anterior mandibular region. Whenever available, CBCT should be utilized as the primary means of radiographic imaging due to its superiority in detecting the presence of the MIC in comparison to traditional radiographs. The presence of the large MIC and its associated neurovascular bundle may contribute to complications such as hemorrhage and sensory disturbance, associated with bone harvesting, implant placement, fracture repair and cystectomy conducted within the interforaminal zone. The incisive branch is a vital anatomical structure that should be accounted for in all procedures conducted within the interforaminal zone.

\section{ORCID}

Jennifer A. Caughey:

https://orcid.org/0000-0003-2868-5470

Quang Do: https://orcid.org/0000-0002-2765-937X

Daniel Shen: https://orcid.org/0000-0002-1990-9308

Hiroe Ohyama: https://orcid.org/0000-0002-3599-1671

Puhan He: https://orcid.org/0000-0002-6135-1053

R. Shane Tubbs: https://orcid.org/0000-0003-1317-1047

Joe Iwanaga: https://orcid.org/0000-0002-8502-7952

\section{Author contributions}

Conceptualization: HO, RST, JI. Data acquisition: JAC, RST, JI. Data analysis or interpretation: JAC, QD, DS. Drafting of the manuscript: JAC, QD, DS, HO. Critical revision of the manuscript: PH, RST, JI. Approval of the final version of the manuscript: all authors.

\section{Conflicts of Interest}

No potential conflict of interest relevant to this article was reported.

\section{Acknowledgements}

The authors sincerely thank those who donated their bodies to science so that anatomical research could be performed. Results from such research can potentially increase mankind's overall knowledge that can then improve patient care. Therefore, these donors and their families deserve our highest gratitude [42].

\section{References}

1. Wadu SG, Penhall B, Townsend GC. Morphological variability of the human inferior alveolar nerve. Clin Anat 1997;10:82-7.

2. Iwanaga J, Matsushita Y, Decater T, Ibaragi S, Tubbs RS. Mandibular canal vs. inferior alveolar canal: evidence-based terminology analysis. Clin Anat 2021;34:209-17.

3. Iwanaga J, Ibaragi S, Takeshita Y, Asaumi J, Horner K, Gest TR, Tubbs RS. Mandibular canal versus inferior alveolar canal: a Delphi study. Clin Anat 2021 Apr 27 [Epub]. https://doi. org/10.1002/ca.23740.

4. Xu Y, Suo N, Tian X, Li F, Zhong G, Liu X, Bao Y, Song T, Tian $\mathrm{H}$. Anatomic study on mental canal and incisive nerve canal in interforaminal region in Chinese population. Surg Radiol Anat 2015;37:585-9.

5. Rodella LF, Buffoli B, Labanca M, Rezzani R. A review of the mandibular and maxillary nerve supplies and their clinical relevance. Arch Oral Biol 2012;57:323-34.

6. Hur MS, Kim HC, Won SY, Hu KS, Song WC, Koh KS, Kim HJ. Topography and spatial fascicular arrangement of the human inferior alveolar nerve. Clin Implant Dent Relat Res 2013;15:88-95.

7. von Arx T, Lozanoff S. Mandibular incisive canal. In: von Arx T, Lozanoff S, editors. Clinical Oral Anatomy: A Comprehensive Review for Dental Practitioners and Researchers. Cham: Springer International Publishing; 2017. p.445-61.

8. Nkenke E, Schultze-Mosgau S, Radespiel-Tröger M, Kloss F, Neukam FW. Morbidity of harvesting of chin grafts: a prospective study. Clin Oral Implants Res 2001;12:495-502.

9. De Andrade E, Otomo-Corgel J, Pucher J, Ranganath KA, St George N Jr. The intraosseous course of the mandibular incisive nerve in the mandibular symphysis. Int J Periodontics Restorative Dent 2001;21:591-7.

10. Krasny A, Krasny N, Prescher A. Study of inferior dental canal and its contents using high-resolution magnetic resonance imaging. Surg Radiol Anat 2012;34:687-93.

11. Yang XW, Zhang FF, Li YH, Wei B, Gong Y. Characteristics of intrabony nerve canals in mandibular interforaminal region by using cone-beam computed tomography and a recommendation of safe zone for implant and bone harvesting. Clin Implant Dent Relat Res 2017;19:530-8.

12. Jacobs R, Lambrichts I, Liang X, Martens W, Mraiwa N, Adriaensens P, Gelan J. Neurovascularization of the anterior jaw bones revisited using high-resolution magnetic resonance 
imaging. Oral Surg Oral Med Oral Pathol Oral Radiol Endod 2007;103:683-93.

13. Hirsch JM, Brånemark PI. Fixture stability and nerve function after transposition and lateralization of the inferior alveolar nerve and fixture installation. Br J Oral Maxillofac Surg 1995;33:276-81.

14. Starkie C, Stewart D. The intra-mandibular course of the inferior dental nerve. J Anat 1931;65(Pt 3):319-23.

15. Stewart D, Wilson SL. Regional anesthesia and innervation of the teeth. Lancet 1928;212:809-11.

16. Mardinger O, Chaushu G, Arensburg B, Taicher S, Kaffe I. Anatomic and radiologic course of the mandibular incisive canal. Surg Radiol Anat 2000;22:157-61.

17. Parnia F, Moslehifard E, Hafezeqoran A, Mahboub F, MojaverKahnamoui H. Characteristics of anatomical landmarks in the mandibular interforaminal region: a cone-beam computed tomography study. Med Oral Patol Oral Cir Bucal 2012;17:e420-5.

18. Gilis S, Dhaene B, Dequanter D, Loeb I. Mandibular incisive canal and lingual foramina characterization by cone-beam computed tomography. Morphologie 2019;103:48-53.

19. Kong N, Hui M, Miao F, Yuan H, Du Y, Chen N. Mandibular incisive canal in Han Chinese using cone beam computed tomography. Int J Oral Maxillofac Surg 2016;45:1142-6.

20. Apostolakis D, Brown JE. The anterior loop of the inferior alveolar nerve: prevalence, measurement of its length and a recommendation for interforaminal implant installation based on cone beam CT imaging. Clin Oral Implants Res 2012;23:1022-30.

21. Uchida Y, Noguchi N, Goto M, Yamashita Y, Hanihara T, Takamori H, Sato I, Kawai T, Yosue T. Measurement of anterior loop length for the mandibular canal and diameter of the mandibular incisive canal to avoid nerve damage when installing endosseous implants in the interforaminal region: a second attempt introducing cone beam computed tomography. J Oral Maxillofac Surg 2009;67:744-50.

22. Pires CA, Bissada NF, Becker JJ, Kanawati A, Landers MA. Mandibular incisive canal: cone beam computed tomography. Clin Implant Dent Relat Res 2012;14:67-73.

23. Sahman H, Sekerci AE, Sisman Y, Payveren M. Assessment of the visibility and characteristics of the mandibular incisive canal: cone beam computed tomography versus panoramic radiography. Int J Oral Maxillofac Implants 2014;29:71-8.

24. Pereira-Maciel P, Tavares-de-Sousa E, Oliveira-Sales MA. The mandibular incisive canal and its anatomical relationships: a cone beam computed tomography study. Med Oral Patol Oral Cir Bucal 2015;20:e723-8.

25. Hunt DR, Jovanovic SA. Autogenous bone harvesting: a chin graft technique for particulate and monocortical bone blocks. Int J Periodontics Restorative Dent 1999;19:165-73.

26. Al-Ani O, Nambiar P, Ha KO, Ngeow WC. Safe zone for bone harvesting from the interforaminal region of the mandible. Clin Oral Implants Res 2013;24 Suppl A100:115-21.

27. Sbordone L, Menchini-Fabris GB, Toti P, Sbordone C, Califano L, Guidetti F. Clinical survey of neurosensory side-effects of mandibular parasymphyseal bone harvesting. Int J Oral Maxil- lofac Surg 2009;38:139-45.

28. Pommer B, Tepper G, Gahleitner A, Zechner W, Watzek G. New safety margins for chin bone harvesting based on the course of the mandibular incisive canal in CT. Clin Oral Implants Res 2008;19:1312-6.

29. Lee CY, Yanagihara LC, Suzuki JB. Brisk, pulsatile bleeding from the anterior mandibular incisive canal during implant surgery: a case report and use of an active hemostatic matrix to terminate acute bleeding. Implant Dent 2012;21:368-73.

30. Mraiwa N, Jacobs R, Moerman P, Lambrichts I, van Steenberghe D, Quirynen M. Presence and course of the incisive canal in the human mandibular interforaminal region: two-dimensional imaging versus anatomical observations. Surg Radiol Anat 2003;25:416-23

31. Kütük N, Demirbaş AE, Gönen ZB, Topan C, Kiliç E, Etöz OA, Alkan A. Anterior mandibular zone safe for implants. J Craniofac Surg 2013;24:e405-8.

32. Romanos GE, Greenstein G. The incisive canal. Considerations during implant placement: case report and literature review. Int J Oral Maxillofac Implants 2009;24:740-5.

33. Wismeijer D, van Waas MA, Vermeeren JI, Kalk W. Patients' perception of sensory disturbances of the mental nerve before and after implant surgery: a prospective study of 110 patients. Br J Oral Maxillofac Surg 1997;35:254-9.

34. Tay AB, Lai JB, Lye KW, Wong WY, Nadkarni NV, Li W, Bautista D. Inferior alveolar nerve injury in trauma-induced mandible fractures. J Oral Maxillofac Surg 2015;73:1328-40.

35. Pickrell BB, Serebrakian AT, Maricevich RS. Mandible fractures. Semin Plast Surg 2017;31:100-7.

36. Ellis E 3rd. A study of 2 bone plating methods for fractures of the mandibular symphysis/body. J Oral Maxillofac Surg 2011;69:1978-87.

37. Agnihotri A, Prabhu S, Thomas S. A comparative analysis of the efficacy of cortical screws as lag screws and miniplates for internal fixation of mandibular symphyseal region fractures: a randomized prospective study. Int J Oral Maxillofac Surg 2014;43:22-8.

38. Sun HH, Lin SC, Park CM, Elo JA. Asymptomatic intrabony radiolucency of the anterior mandible. Oral Surg Oral Med Oral Pathol Oral Radiol 2020;130:350-6.

39. Bilginaylar K, Orhan K, Uyanik LO. Mandibular incisive canal in relation to periapical surgery. Contemp Clin Dent 2016;7:79-81.

40. Perkins D, Stiharu TI, Swift JQ, Dao TV, Mainville GN. Intraosseous schwannoma of the jaws: an updated review of the literature and report of 2 new cases affecting the mandible. J Oral Maxillofac Surg 2018;76:1226-47.

41. Rengaswamy V. Central neurilemmoma of the jaws. Review of literature and case report. Int J Oral Surg 1978;7:300-4.

42. Iwanaga J, Singh V, Ohtsuka A, Hwang Y, Kim HJ, Moryś J, Ravi KS, Ribatti D, Trainor PA, Sañudo JR, Apaydin N, Şengül G, Albertine KH, Walocha JA, Loukas M, Duparc F, Paulsen F, Del Sol M, Adds P, Hegazy A, Tubbs RS. Acknowledging the use of human cadaveric tissues in research papers: recommendations from anatomical journal editors. Clin Anat 2021;34:2-4. 